\title{
The determinants of methadone maintenance treatment in opioids dependence users: A survival analysis study
}

\author{
Mohammad Aram Ahmadi ${ }^{1}$, Ghobad Moradi ${ }^{1}$, Sahar khoshravesh², Daem Roshani*1(D) Naseh Qaderi ${ }^{3}$, \\ Mohiadin Amjadian ${ }^{4}$
}

Received: 21 Nov 2018

Published: 4 Nov 2020

\begin{abstract}
Background: Staying in treatment is one of the basic principles in maintenance treatment with methadone and it is considered as a success criterion in the treatment procedure. This study aimed at analyzing effective determinants on staying in treatment in methadone treatment procedure.

Methods: This was a retrospective cohort study, in which 6 MMT centers were randomly selected to be studied. The data were collected using the patients' medical records. Therefore, 1008 medical record files belonging to the patients who received methadone treatment from April 2013 to August 2017 were investigated. Proportional hazard Cox regression (extended) was used to specify the determinants of the methadone maintenance treatment and STATA 11 was used for data analysis.

Results: The patients' mean age was 38.8 years $( \pm 1.08)$, and $75.8 \%$ were married and only $39.1 \%$ had full-time jobs. The mean age for the first substance abuse was at 24.31years $( \pm 7.93)$. The average time of staying in the treatment was 28.8 months. The results of the adjusted Cox proportional hazards regression showed among the 14 variables entered into the model, in the 1-12-month interval age of first drug use $(\mathrm{HR}=0.945,95 \% \mathrm{CI}=0.908-0.983, \mathrm{p}=0.005)$, specific drug use $(\mathrm{HR}=1.14,95 \% \mathrm{CI}=1.026-1.268, \mathrm{p}=0.014)$, and the frequency of treatment $(\mathrm{HR}=0.974,95 \% \mathrm{CI}=0.959-0.990, \mathrm{p}=0.002)$ were significantly correlated with survival status. The 13-36month interval drug use $(\mathrm{HR}=0.931,95 \% \mathrm{CI}=0.886-0.978, \mathrm{p}=0.005)$ was significantly correlated with survival status. Also, the range of 37-53 month drug use $(\mathrm{HR}=1.058,95 \% \mathrm{CI}=1.001-1.119, \mathrm{p}=.044)$ had a significant relationship with survival status.

Conclusion: This study showed age of first drug use, specific drug use, the frequency of treatment and drug use were correlated with a decrease in staying duration in treatment. Therefore, taking these factors into consideration in designing and administering various interventions in addiction treatment and consulting centers is of paramount importance.
\end{abstract}

Keywords: Maintenance, Models, Opioid users, Relapse, Methadone

Conflicts of Interest: None declared

Funding: Research Deputy and Health Social Factor Research Center of Kurdistan University of Medical Sciences

*This work has been published under CC BY-NC-SA 1.0 license.

Copyright $\odot$ Iran University of Medical Sciences

Cite this article as: Ahmadi MA, Moradi G, khoshravesh S, Roshani D, Qaderi N, Amjadian M. The determinants of methadone maintenance treatment in opioids dependence users: A survival analysis study. Med J Islam Repub Iran. 2020 (4 Nov);34:149. https://doi.org/10.47176/mjiri.34.149

\section{Introduction}

In 2017, an estimated 271 million people, or $5.5 \%$ of the global population aged 15-64 years had used drugs in the previous year, while 35 million people are estimated to be

Corresponding author: Dr Daem Roshani, d.roshani@muk.ac.ir

1. Social Determinants of Health Research Center, Research Institute for Health Development, Kurdistan University of Medical Sciences, Sanandaj, Iran

2. Students Research Committee, Hamadan University of Medical sciences, Hamadan, Iran

3. Department of Health Education \& Promotion, School of Medical Sciences, Tarbiat Modares University, Tehran, Iran

4. Department of Clinical Psychology, Kurdistan University of Medical Sciences, Sanandaj, Iran suffering from drug use disorders (1). One of the most important characteristics of substance abuse disorders is frequent attempts for treatment and repeated relapses (2).

$\uparrow$ What is "already known" in this topic:

Little is known about influential factors on staying length in MMT treatment in Iran, and the available data have been gathered based on small samples in some of the studies; therefore, the aim of this study was to survey the effective determinants on staying in treatment in methadone treatment procedure.

$\rightarrow$ What this article adds:

These results show the age of substance abuse onset and the number of treatments have a significant relationship with staying status in methadone treatment. 
Due to high number of relapses and an increase in deadly poisonings after detoxification, methadone maintenance treatment (MMT) is considered as first-line treatment in substance-dependent patients in most of the countries in the world $(3,4)$. The results of some studies show MMT, in addition to a decrease in craving for substance use, causes a decrease in number of crimes related to substance abuse and transmission of diseases such as hepatitis and HIV (5-7). Iran is one of the countries with a high number of crimes related to opioids use (8), and it seems that methadone maintenance treatment for these patients has attracted a special attention in developing countries. In fact, a study in Iran showed the longest periods of being away from substance abuse and the longest periods of staying in the treatment procedure among substance abusers, belonged to those who took MMT compared to other treatments (9).

MMT has been widely used in Iran since 2004 and foundations and facilities for presenting MMT services have been prepared since then, so that there are 1400 MMT centers throughout the country now (10). Although MMT is used extensively in different countries, some challenges are involved in its use. One of the most important challenges facing MMT is that the patients do not stay in the treatment much and they experience high rates of relapse (11).

Since staying length in the treatment in MMT has a positive correlation with other positive consequences of MMT, such as decrease in relapse risk (12), staying in the treatment is one of the basic principles in MMT and it is considered as a criterion for the treatment success (13).

Little is known about influential factors on staying length in MMT treatment in Iran, and the available data have been gathered based on small samples (14). Moreover, the patients' follow-up periods were short as well (15). Therefore, studying these factors in larger samples and with longer follow-up periods is needed to determine effective modalities which might enhance MMT efficacy. In addition to filling the existing data gap in the related studies, this will increase MMT outcomes generalizability. This study aimed to investigate influential determinants of the staying length in methadone treatment.

\section{Methods}

This was a retrospective cohort study in which 6 MMT centers were randomly selected among 58 centers in Sanandaj, West of Iran. The medical data of all patients were extracted from their medical records using census report method.

In fact, the medical records of 1008 patients who received methadone maintenance treatment from 2013 to 2017 were studied. The patients who took other treating methods such as buprenorphine were excluded from the study. To avoid ethical problems, the names of the patients were coded. All variables were recorded based on the patients' registration data for the study and their initial interview, and did not show the patients' final medical status. All data, including demographic variables (age, sex, education status, marital status, employment, and housing status), first time substance abuse age, history of substance abuse, other kinds of substance abuse, the number of treatments, history of mental disorders, history of having any specific disease, history of taking any kind of medications for any reason, and any criminal records were extracted as predictive variables through a check list. Moreover, staying length in methadone treatment, as a response variable, was coded one (1) for those patients who received MMT in the time of the study and zero (0) for those who had left the treatment before the study was over (August 2017). In case of patients' incomplete medical information, the needed data were gathered through an in-person interview or a phone call. The time length of the study as a variable was defined (time response) as the time from patients' registration in the study to their last visit by the researcher (at least 1 month and at most 53 months).

After dividing the 53-month interval into 3 subgroups of less than 1 year (1-12 months), 1 to 3 years (13-36 months) and over 3 years (53-37 months), the test of relative risk hypothesis was tested and age, education, the time coefficients, drug use history, specific drug use, and number of treatments were added to the model as a coefficient of time in the generalized Cox regression analysis.

Survival model is one of the methods for analyzing disease durability or relapse. These models have 2 advantages over classic regression models: 1) They are able to investigate censored data in which the studied event does not happen throughout the time of the study at least for a part of the studied sample. 2) They are able to determine effective factors on the survival time interval until the event occurrence in the time range of the study. Semiparametric proportional hazard Cox regression is one of the most common and a suitable model in survival analysis, and it is not based on any specific distribution for survival times. Therefore, it is a proper substitute for parametric survival models in case of having fitness of hazards for all independent variables in the model (16). To analyze the data, the Cox regression model was fitted to investigate any possible relationship between dependent and independent variables in the study. On the other hand, extended proportional hazard Cox regression was used to determine factors affecting staying in treatment with methadone. After fitting Cox regression model, significant variables in the model were investigated through proportional hazard Cox regression goodness of fit (based on Sheffield residuals tests). Since hazard ratios (HR) for age, education status, history of substance abuse, history of taking any kind of medication for any reason, and the number of treatments were not stable through the time, they were entered into the model as a multiplication in the time of the study and modeling was done using extended proportional hazard Cox method. STATA 13 was used for data analysis and significant level was set at 0.05 for all tests.

\section{Results}

The average age of the patients was $38.8 \pm 1.08$ years based on their medical records and the most frequent age group was 36-55 years group $(n=492,48.8 \%)$. Also, 962 patients were men (95.4\%) and the most frequent education group was junior high school $(n=267,26.5 \%)$. More- 
over, $764(75.8 \%)$ patients were married. Detailed demographic data of the patients are presented in Table 1. Since survival was defined as staying in the treatment with methadone, $734(72.8 \%)$ patients stayed in the treatment and $274(27.2 \%)$ left the treatment in the time of the study. The median of staying time in treatment was 28 months, indicating that $50 \%$ of the patients were in the treatment procedure for 28 months and its average time was 28.8 months. The results of the goodness of fit test for proportional hazards hypothesis with 5 significant variables were given in Table 2 . After identifying 5 variables that were found to violate the relative hazard hypothesis, the regression model of Cox relative risks was fitted to the data based on the extended state, and the results were presented in Table 3. To describe the survival status, Kaplan-Meier survival chart was plotted (Fig. 1). The results of extended proportional hazard Cox regression model analysis showed among 14 entered variables into the model in the first year the followings occurred:

1) Age of onset of substance abuse: It ( $\mathrm{HR}=0.945$, $\mathrm{CI}=0.908,0.983$ ) showed for one-year increase in onset of substance abuse there was $5.8 \%$ decrease in the probabil- ity of staying in treatment $(\mathrm{p}=0.005)$.

2) History of taking any kind of medication for any reason: It $(\mathrm{HR}=1.14, \mathrm{CI}=1.026,1.268)$ showed that taking any kind of medication for any reason had $14 \%$ increase in probability of staying in the treatment $(\mathrm{p}=0.014)$.

3) Treatment number as a variable showed ( $\mathrm{HR}=0.974$, $\mathrm{CI}=0.96,0.99$ ), for one time increase in treatment numbers there was $2.6 \%$ decrease in the probability of staying in the treatment $(\mathrm{p}=0.002)$.

Between first and third years other kinds of substance abuse were as follow: It $(\mathrm{HR}=0.931, \mathrm{CI}=0.886,0.978)$ showed other kinds of substance abuse had $7.4 \%$ decrease in the probability of staying in the treatment $(\mathrm{p}=0.005)$.

The results for more than three year:

1) Employment status: Having considered "full-time job" status as a base level, casual part-time job status $(\mathrm{HR}=0.94, \mathrm{CI}=0.891,0.992)$ had $6.4 \%$ lower probability of staying in the treatment compared to the full-time job status $(\mathrm{p}=0.026)$.

2) Other kinds of substance abuse: It $(\mathrm{HR}=1.058$, $\mathrm{CI}=1.001,1.119)$ showed other kinds of substance abuse had $5.8 \%$ increase in the probability of staying in the

Table 1. Sociodemographic characteristics of the study sample $(\mathrm{n}=1008)$

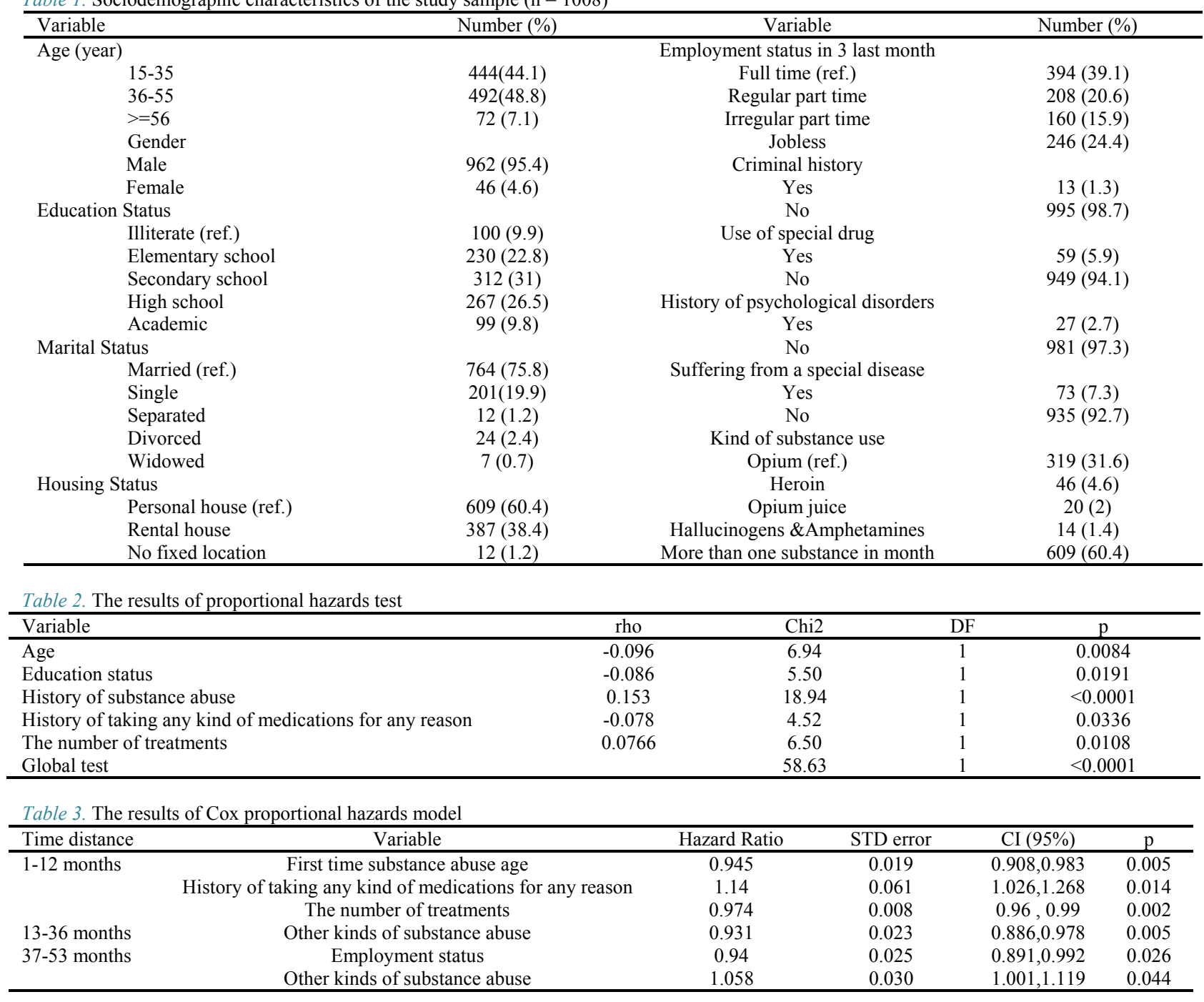




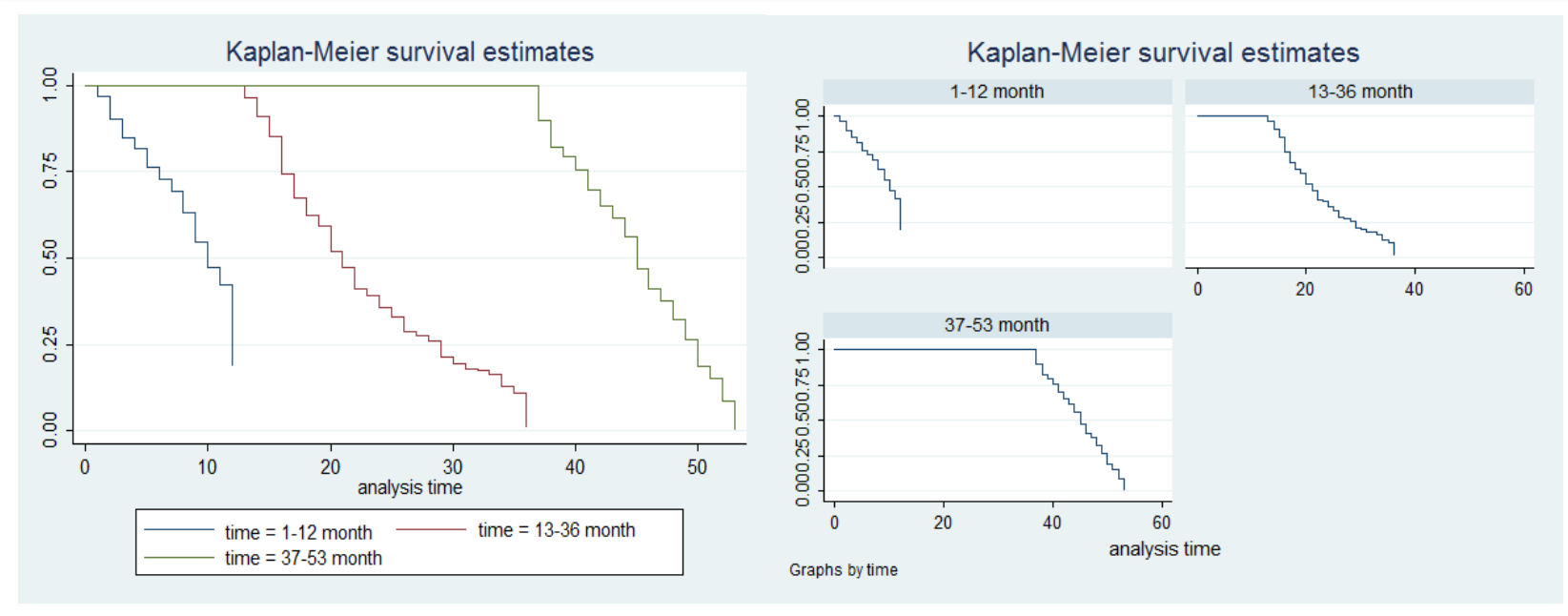

Fig. 1. Chart survival (retention time)

treatment $(\mathrm{p}=0.044)$ (Table 3).

Moreover, age, sex, education status, housing, marital status, substance abuse history, mental disorder history, specific disease history, and crime records had no significant relationship with staying in methadone treatment.

\section{Discussion}

This study tried to investigate the influential determinants on staying time length in treatment with methadone in substance abusers. The results of analysis showed the average staying time was 28.8 months and its median was 28 months as well for all times. This was not in line with the results of Pashaee and Mohebi's' studies in which the staying time median in the treatment was 6 months, and $75 \%$ of the patients had a staying time length of 26 months and the average staying time length in the treatment was 5.5 months, respectively $(10,14)$. Moreover, Sarssvita's study in Indonesia showed $61.3 \%$ of the patients stayed 3 months the treatment and $74.2 \%$ stayed 6 months (17). In addition, another study in the USA showed 3 months of stay in the treatment for the patients (18).

It seems that one of the possible reasons for these contradictory results lies in the difference in their follow-up time lengths, so that this time was 53 months in this study, but it was 6 months or less in the mentioned studies (10, $18,19)$. In fact, when the treatment follow-up length is short, most of the patients' experience relapses and they leave the treatment after the follow-up. Therefore, it causes a decrease in the staying time in the treatment among the patients. Other probable reasons of difference in staying in the treatments in various studies might be due to the differences in sample size, and social, economic and cultural characteristics of the samples, and intervention qualities of addiction treatment clinics.

Single patients had lower staying time lengths in the treatment in this study which is in line with Hosseini and weis' studies in Iran and China, respectively $(20,21)$. In fact, it is proposed that marriage or living with parents seem to act as a protective factor against patients' relapses that were treated with methadone. Marriage creates sense of solidarity and increases sense of responsibility in the patients which leads to an increase in their family protection and prevents relapses among them (22). It seems the main reason of such a difference between married and nonmarried patients is the amount of social protection they might receive, as the married receive social protection much more because of their larger social networks and relations compared to those single or living alone. Since the treatment period is very long in substance abuse patients and they need family and social protection, economical and emotional family protections seem to play a great role in the methadone maintenance treatment success $(23,24)$. Those unemployed patients who have income had lower staying time lengths in the treatment compared to those with full-time jobs. Unemployment increase susceptibility to leaving the treatment procedure and restarting the substance abuse among the patients $(25,26)$. In fact, those unemployed patients, due to having more freetime, spending more time at home, and increasing conflicts with family numbers, are more prone to restarting substance abuse and leaving the treatment procedure as well (25). Moreover, if these patients themselves have incomes and have no financial constraints to buy the substances they use, this might act like a motivational factor which triggers relapses and treatment leaving among them.

In addition, the study showed those patients who initiated substance abuse in younger ages had lower staying time lengths in the treatment. This is in line with the results of some other studies which claim that the patients who started substance abuse later in their life had a better prognosis and higher staying time lengths in the treatment compared to those who started substances abuse sooner (27). It seems psychological characteristics of those younger substance abusers play a great role here. In fact, patients' impulsive and agitated behavior may make them prone to quit treatment and cause relapse. On the other hand, those who initiated substance abuse later in their lives had shorter substance dependence periods and less severe addictions; therefore, they had higher staying time lengths in the treatment procedure. The results of Fox (28) 
and Guindalini (29) showed these patients with longer substance dependence periods and more severe addictions had more craving for substance abuse, which led to treatment failure (30). The patients with casual part-time jobs had got higher treatment maintenance time length compared to those with full-time jobs. There seems to be contradictory results about the effects of having jobs and unemployment on treatment maintenance in various studies done in this area. For example, a study by Shah in Baltimore showed sociodemographic factors, including having or not having jobs, might affect the treatment maintenance (31), but 2 other studies did not show any significant effect $(14,15)$. Those with casual part-time jobs seemed not to have time constraints to attend treatment sessions, which might have caused better treatment outcomes and higher staying time in the treatment. Moreover, those patients who had higher numbers of treatment stayed more in the treatment; this is in line with the results of some other studies $(32,33)$. However, some evidences show the history of addiction abandonment and increase in the times of treatment have a negative effect on staying time length in the treatment $(14,18)(22)$. The results of some other studies showed those who received methadone treatment, resisted more against substance abuse cravings (34), and this might be a possible reason for their longer stay in the treatment.However, large sample size, interview with some of the patients about how they took methadone (tablets or syrup), and calculating odds ratio for influential variables on staying time length in the treatment were the strong points of this study. However, there were some limitations. Some variables were excluded from the study due to not being filled in the required data by the patients or lack of access to the patients. In addition, due to the patients' social status considerations, some MMT centers' lack of cooperation and lack of legal license to follow-up their patients' treatment after the treatment period, the recorded data of the patients throughout the treatment period from the selected MMT centers were used here. Therefore, studying the staying time length in the treatment or treatment leaving variables were limited to those centers.

\section{Conclusion}

This study showed the age of first drug use, specific drug use, the frequency of treatment, and drug use were correlated with a decrease in staying duration in treatment. Casual higher number of treatments was correlated with higher staying time in the treatment with methadone. Thus, paying enough attention to these factors in designing and using treatment interventions for such patients is highly important to enhance the efficacy of such interventions in MMT centers.

\section{Acknowledgment}

This study was done using funds from the Research Deputy and Health Social Factor Research Center of Kurdistan University of Medical Sciences. We gratefully thank them and also all MMT centers staff and patients.
Conflict of Interests

The authors declare that they have no competing interests.

\section{References}

1.UNODC. http://www.unodc.org/westandcentralafrica/en/2019_06_2 5_world_drug_day.html

2. Krenek M, Maisto S. Life events and treatment outcomes among individuals with substance use disorders: a narrative review. Clin Psychol Rev. 2013;33(3):470-83.

3. O'Connor P. Methods of detoxification and their role in treating patients with opioid dependence. JAMA. 2005;294:961-3.

4. Watson B, Lingford-Hughes A. Pharmacological treatment of addiction. Psychiatry. 2007;6(7): 309-12.

5. Hedrich D, Alves P, Farrell M, Stöver H, Møller L, Mayet S. The effectiveness of opioid maintenance treatment in prison settings: a systematic review. Addiction. 2012;107:501-17

6. Kerr T, Marsh D, Li K. Factors associated with methadone maintenance therapy use among a cohort of polysubstance using injection drug users in Vancouver. Drug Alcohol Depend. 2005;80:329-35.

7. Schwartz RP, Highfield DA, Jaffe JH. A randomized controlled trial of interim methadone maintenance. Arch Gen Psychiatr. 2006;63:102-9.

8. United Nations Office on Drug and Crime. World Drug Report 2011. 2012.

9. Moridi M, Khoshravesh S, Noori R, Pashaei T. The survey of relapse's styles among drug users and stimulants. Pajouhan Sci J. 2017;15(3):10-5.

10. Mohebbi MD, Sargolzei N, Adibi A. Evaluation of retention in methadone treatment in patients attending Baharan hospital clinic in Zahedan city. Sci J Hamdan Univ Med Sci. 2015;22(1):30-6.

11. Hoseini F, Yaseni Ardakani S, Ahmadieh M, Vafainasab M, Kholasezade G, Dastjerdi G. The survival rate on methadone maintenance treatment. J Shaheed Sadoughi Univ Med Sci. 2010;18(3):152-8.

12. Strike C, Gnam W, Urbanoski K, Fischer B, Marsh D, Millson M. Factors predicting 2- year retention in methadone maintenance treatment for opioid dependence. Addict Behav. 2005;30(5):1025-8.

13. Fareed A, Casarella J, Amar R, Vayalapalli S, Drexler K. Benefits of retention in methadone maintenance and chronic medical conditions as risk factors for premature death among older heroin addicts. J Psychiatr Pract. 2009;15(3): 227-34.

14. Pashaei T, Moeeni M, Roshanaei Moghdam B, Heydari H, Turner NE, Razaghi EM. Predictors of treatment retention in a major methadone maintenance treatment program in iran: a survival analysis. J Res Health Sci. 2014;14(4):291-5.

15. Sheikh Fathollahi M, Torkashvand F, Najmeddin H, Rezaeian M. Predictors of One-Year Retention in Methadone Maintenance Treatment (MMT) in Iran, Rafsanjan. Int J High Risk Behav Addict. 2016;5(3):e29121.

16. Moeeni M, Razaghi EM, Mahmoudi M, Pashaei T. Analysis of retention time among a sample of opioid dependents participating in the Methadone Maintenance Treatment Program in Iran. J School Public Health Institute Public Health Res. 2013;11(3).

17. Srivastava A, Kahan M, Ross S. The effect of methadone maintenance treatment on alcohol consumption: A systematic review. J Subst Abuse Treat. 2008;34(2):215-23.

18. Johnson RE, Chutuape MA, Strain EC. A comparison of levomethadyl acetate, buprenorphine, and methadone for opioids dependence. N Engl J Med. 2000;343:1290-7.

19. Sarasvita R, Tonkin A, Utomo B, Ali R. Predictive factors for treatment retention in methadone programs in Indonesia. J Subst Abuse Treat. 2012;42(3):239-46.

20. Hosseini F, Yassini M, Ahmadieh M, Vafaei-Nasab M, Kholasezadeh G, Dastjerdi G. High risk behavior conference. Shaheed Sadoughi Univ Med Sci. 2010. 2010;18(3):152-8.

21. Wei X, Wang L, Wang X, Li J, Li H, Jia W. A study of 6-year retention in methadone maintenance treatment among opioiddependent patients in Xi'an. J Addict Med. 2013;7(5):342-8.

22. Babaeian N, Maarefvand M, Hosseinzadeh S. The effectiveness of working in vocational network of recovered on substance users' retention in treatment. J Soc Work. 2016;5(3):51-61. 
23. Yang F, Lin P, Li Y, He Q, Long Q, Fu X, et al. Predictors of retention in communitybased methadone maintenance treatment program in Pearl River Delta, China. Harm Reduct J. 2013;10(3).

24. Zhou K, Zhuang G. Retention in methadone maintenance treatment in mainland China, 2004-2012: A literature review. Addict Behav J. 2014;39:22-9.

25. Mokri A. Instructions for psychoactive drug abuse treatment based on modified matrix pattern. Ministry of Health and Medical Education, Office of Mental Health, Social and addiction. 2011.

26. Hajian K, Khirkhah F, Falatoni M. Epidemiology of Addiction among Volunteered Addicts Attending in Detoxification Centers. J Guilan Uni Med Sci. 2013;22(87):22-30.

27. Sayyadi Anari AR, Esmaili A, Nazer M, Khaleghi E. Demographic Features of Opium Dependent Patients with Successful Withdrawal Attempts at RUMS Outpatient Clinic. Iran J Psychiatry Clin Psychol. 2002;8(1):29-38.

28. Fox HC, Talih M, Malison R, Anderson GM, Kreek MJ. Frequency of recent cocaine and alcohol use affects craving and associated responses to stress and drug-related cues. Psychoneuroendocrinology. 2005;30:880-91.

29. Guindalini C, Wallada H, Breen G, Laranjeira R. Concurrent crack and powder cocaine users from Sao Paulo: Do they represent a different group? BMC Public Health. 2006;6(10).

30. Walton-Moss B, McCaul ME. Factors associated with lifetime history of drug treatment among substance dependent women. Addict Behav J. 2006;31:246-53

31. Shah NG, Galai N, Celentano DD, Vlahov D, Strathdee SA Longitudinal predictors of injection cessation and subsequent relapse among a cohort of injection drug users in Baltimore, MD, 19882000. Drug Alcohol Depend J. 2006;83(2):147-56.

32. Dennis ML, Scott CK, Funk R, Foss MA. The duration and correlates of addiction and treatment careers. J Subst Abuse Treat. 2005;28 Suppl 1:S51-62.

33. Hser YI, Grella CE, Hsieh SC, Anglin MD, Brown BS. Prior treatment experience related to process and outcomes in DATOS. Drug Alcohol Depend. 1999;57(2):137-50.

34. Chehri A, Mirzaei F, Khazaei H, Rezaei O, Ekhtiari H, h Mokri A, et al. Comparison of craving for opioid in opioid-dependent individuals and people under methadone maintenance treatment. J Kermanshah Uni Med Sci. 2014;17(11): 718-726. 